\title{
Modelo Dinâmico Simplificado para um Sistema Encanamento-Riser-Separador sob Regime de Fluxo com Golfadas ${ }^{1}$
}

A. SAUSEN ${ }^{2}$, P.R. BARROS 3 , Departamento de Engenharia Elétrica, Universidade Federal de Campina Grande, UFCG, 58109-900 Campina Grande, PB, Brasil.

\begin{abstract}
Resumo. Neste artigo é apresentado o desenvolvimento de um modelo dinâmico simplificado para um sistema encanamento-riser-separador sob regime de fluxo com golfadas. Esse tipo de escoamento caracteriza-se por fluxo severo e irregular com oscilações de pressão e vazão na tubulação e nas unidades de separação em indústrias de produção de petróleo. O modelo obtido é resultado do acoplamento entre o modelo dinâmico simplificado para o sistema encanamento-riser sujeito ao regime de fluxo com golfadas, e o modelo de um separador cilíndrico horizontal bifásico. São apresentados os resultados das simulações e observa-se que o modelo proposto representa de forma satisfatória a dinâmica da golfada no sistema encanamentoriser, bem como dentro do separador.
\end{abstract}

Palavras-chave. Modelagem Matemática, Sistema Encanamento-Riser-Separador, Golfadas.

\section{Introdução}

A golfada é um regime de fluxo multifásico que forma-se dentro das tubulações, que unem os poços no fundo do oceano às plataformas de produção na superfície, nas indústrias de produção de petróleo. Caracteriza-se por fluxo severo e irregular com oscilações de pressão e vazão em todo o processo de produção. Storkaas [8] classifica as golfadas que ocorrem em diferentes partes do sistema encanamento-riser (tubulação ascendente dentro do oceano) em quatro tipos: golfadas hidrodinâmicas, golfadas no riser, golfadas transientes, e golfadas em terrenos. Nesse trabalho será abordada a golfada no riser que combinada ou iniciada com a golfada em terrenos é uma das maiores instabilidades em sistemas encanamento-riser-separador na produção de petróleo $[6,7,8]$.

É apresentada na Figura 1 o comportamento cíclico da golfada no riser que é dividido em quatro fases:

1. Formação: a golfada inicia-se com baixa pressão e velocidade dos fluidos na seção de alimentação do encanamento, então em um determinado momento o líquido bloqueia o fluxo de gás no ponto-baixo iniciando a formação de uma golfada de líquido contínua no riser.

\footnotetext{
${ }^{1}$ Apoio financeiro CNPq.

2 airam@dee.ufcg.edu.br, bolsista de doutorado CNPq.

3 prbarros@dee.ufcg.edu.br.
} 


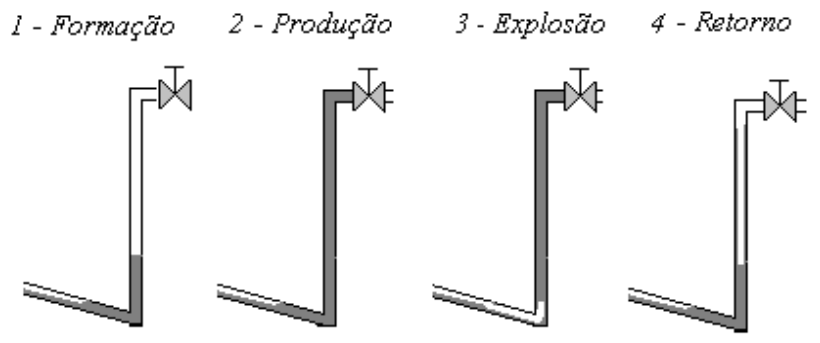

Figura 1: Descrição do ciclo da golfada no riser.

2. Produção: enquanto a quantidade de líquido no riser aumenta mais rapidamente que a variação da pressão entre o topo e a seção de alimentação, a golfada continua crescendo.

3. Explosão: ocorre quando a pressão do gás na seção de alimentação tornase maior que o peso da coluna de líquido no riser, conseqüentemente essa quantidade de líquido é enviada para fora do sistema.

4. Retorno: após uma grande quantidade de líquido e de gás deixarem o sistema, a pressão na seção de alimentação diminui, então o líquido começa a acumular no ponto-baixo reiniciando o ciclo.

A golfada gera conseqüências indesejadas em todo o processo de produção de petróleo tais como, períodos com nenhuma produção de líquido, seguido de períodos com grande produção dentro do separador, causando redução na capacidade de produção, parada de emergência na plataforma devido ao alto nível de líquido nos separadores, inundação quando for muito severa, corrosão e desgaste nos equipamentos do processo, altos custos com manutenção. Um, ou todos esses problemas causam diferentes prejuízos, o principal é de ordem econômica em decorrência da redução na capacidade de produção de petróleo. Por esses motivos é necessária a aplicação de estratégias que evitam a golfada em sistemas encanamento-riser, ou minimizam a mesma nas unidades de separação. Pois com um fluxo estável nos instrumentos à jusante da válvula no topo do riser é possível melhorar a regularidade e o desempenho do processo, e o mais importante acelerar e aumentar a produção de petróleo.

Neste contexto o desenvolvimento de um modelo matemático que representa de forma satisfatória a dinâmica da golfada no sistema encanamento-riser-separador torna-se necessário. Na literatura são encontrados modelos que representam a dinâmica do fluxo com golfadas no sistema encanamento-riser $[2,3,7,8]$, porém não mencionam a dinâmica da mesma no separador. Nos poucos trabalhos que consideram um separador $[1,4]$ sua modelagem não é apresentada e a dinâmica da golfada é representada pela função senóide.

Nesse artigo é desenvolvido um modelo matemático para um sistema encanamento - riser - separador sujeito ao regime de fluxo com golfadas e adequado para as aplicações de controle. O restante do artigo está organizado da seguinte forma. $\mathrm{Na}$ Seção 2. são apresentadas as hipóteses da modelagem e as equações do modelo matemático para um sistema encanamento-riser-separador. Na Seção 3. são apresentados os resultados das simulações do modelo em malha aberta. E por fim, na Seção 4. são apresentadas as conclusões. 


\section{O Modelo Matemático}

Nessa Seção é proposto um modelo matemático para um sistema encanamento-riserseparador com fluxo bifásico (i.e., gás-líquido) resultante do acoplamento entre o modelo dinâmico simplificado de Storkaas [8] para o sistema encanamento-riser em condições de golfadas, e o modelo de um separador cilíndrico horizontal bifásico apresentado em Sausen e Barros [5]. O novo modelo é denominado modelo dinâmico simplificado para um sistema encanamento-riser-separador. A seguir são apresentadas as hipóteses da modelagem, as equações do modelo, e por fim como ocorre o escoamento de líquido e de gás dentro da tubulação para o separador.

\subsection{Hipóteses do modelo}

Na Figura 2 é apresentada uma ilustração do sistema encanamento-riser-separador, e as hipóteses de modelagem são enunciadas a seguir.

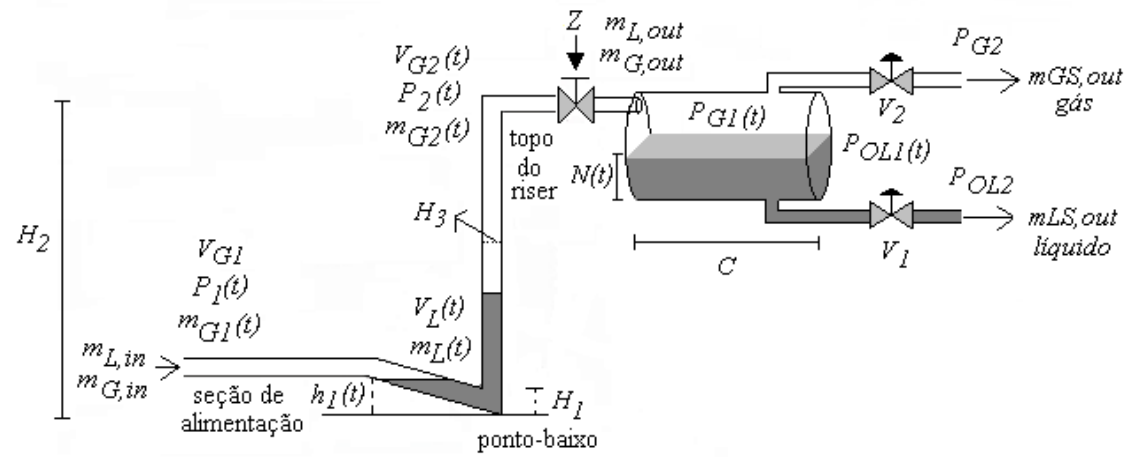

Figura 2: Ilustração do sistema encanamento-riser-separador com a golfada em formação.

A1: Negligenciam-se as dinâmicas do nível de líquido na seção de alimentação ou seja, a velocidade e a alimentação de líquido, e o volume de gás na seção de alimentação do encanamento são constantes.

A2: Há somente um estado dinâmico de líquido $m_{L}(t)$ no ponto-baixo, que inclui o declive do encanamento e uma porção de líquido no riser.

A3: Há dois estados dinâmicos de gás $m_{G 1}(t)$ e $m_{G 2}(t)$, ocupando os volumes $V_{G 1}$ e $V_{G 2}(t)$ na tubulação, separados pelo ponto-baixo, e conectados por uma relação de fluxo e pressão.

A4: A mistura do fluxo mássico de líquido e de gás que saem do sistema no topo do riser é representada através da equação de uma válvula simplificada.

A5: O balanço de pressão entre a seção de alimentação do encanamento $P_{1}(t)$ e o topo do riser $P_{2}(t)$ é estacionário.

A6: Não ocorre reação química entre os fluidos.

A7: Cada um dos fluidos consiste de um componente único dentro do separador. 
A8: Negligencia-se a porção de líquido misturada ao gás na entrada do separador.

A9: Representam-se respectivamente os fluxos mássicos de líquido e de gás que saem do separador através da equação de uma válvula simplificada.

A10: O líquido é incompressível.

A11: A temperatura do sistema é constante.

A12: O comportamento do gás é ideal.

\subsection{Equações do modelo}

O modelo dinâmico simplificado para um sistema encanamento-riser-separador é formado por um conjunto de equações diferenciais não-lineares baseado nas equações de conservação de massa do sistema, onde as equações (2.1)-(2.3) representam a dinâmica do sistema encanamento-riser, e as equações (2.4)-(2.5) representam a dinâmica do separador:

$$
\begin{gathered}
\dot{m}_{L}(t)=m_{L, \text { in }}-m_{L, \text { out }}(t) \\
\dot{m}_{G 1}(t)=m_{G, \text { in }}-m_{\text {Gint }}(t) \\
\dot{m}_{G 2}(t)=m_{G 1}(t)-m_{G, \text { out }}(t) \\
\dot{N}(t)=\frac{\sqrt{r_{s}^{2}-\left(r_{s}-N(t)\right)^{2}}}{2 C \rho_{L} N(t)\left[3 r_{s}-2 N(t)\right]}\left[m_{L, \text { out }}(t)-m_{L S, \text { out }}(t)\right] \\
\dot{P}_{G 1}(t)=\frac{\rho_{L} \Phi\left[m_{G, \text { out }}(t)-m_{G S, \text { out }}(t)\right]+P_{G 1}(t)\left[m_{L, \text { out }}(t)-m_{L S, \text { out }}(t)\right]}{\rho_{L}\left[V_{S}-V_{L S}(t)\right]} .
\end{gathered}
$$

onde: $m_{L}(t)$ é a massa de líquido no ponto-baixo da tubulação em $k g ; m_{G 1}(t)$ é a massa de gás na seção de alimentação do encanamento (i.e., volume $V_{G 1}$ ) em $k g$, $m_{G 2}(t)$ é a massa de gás no topo do riser (i.e., volume $\left.V_{G 2}(t)\right)$ em $k g, N(t)$ é o nível de líquido dentro do separador em $m$, e $P_{G 1}(t)$ é a pressão do gás dentro do separador em $N / m^{2} ; \dot{m}_{L}(t), \dot{m}_{G 1}(t), \dot{m}_{G 2}(t), \dot{N}(t)$, e $\dot{P}_{G 1}(t)$ são respectivamente suas derivadas em relação ao tempo; $m_{L, \text { in }}$ e $m_{G \text {, in }}$ são os fluxos mássicos de líquido e gás que entram na seção de alimentação do encanamento em $\mathrm{kg} / \mathrm{s} ; m_{L, \text { out }}(t)$ e $m_{G \text {,out }}(t)$ são os fluxos mássicos de líquido e gás que saem através da válvula $\mathrm{Z}$ no topo do riser e por conseguinte entram no separador em $\mathrm{kg} / \mathrm{s} ; m_{\text {Gint }}(t)$ é o fluxo mássico de gás interno na tubulação que flui do volume $V_{G 1}$ para o volume $V_{G 2}(t)$ em $\mathrm{kg} / \mathrm{s} ; m_{L S \text {,out }}(t)$ é o fluxo mássico de líquido que sai através da válvula $V_{1}$ do separador em $\mathrm{kg} / \mathrm{s} ; m_{G S, \text { out }}(t)$ é o fluxo mássico de gás que sai através da válvula $V_{2}$ do separador em $\mathrm{kg} / \mathrm{s} ; r_{s}$ é o raio do separador em $m$; $C$ é o comprimento do separador em $m ; \rho_{L}$ é a densidade de líquido em $\mathrm{kg} / \mathrm{m}^{3} ; V_{S}$ é o volume do separador em $m^{3} ; V_{L S}(t)$ é o volume de líquido no separador em $m^{3} ; \Phi=\frac{R T}{M_{G}}$ é uma constante; $R$ é a constante universal do gases ideais $\left(8314 \frac{J}{K \cdot k m o l}\right)$; $T$ é a temperatura no sistema em $K ; M_{G}$ é o peso molecular do gás em $\mathrm{kg} / \mathrm{kmol}$.

O balanço de pressão estacionário no sistema encanamento-riser é dado pela variação entre a pressão na seção de alimentação do encanamento $\left(P_{1}(t)\right)$ e a pressão no topo do riser $\left(P_{2}(t)\right)$. Esse balanço é igual à diferença entre as pressões 
hidrostáticas dos fluidos no riser e no declive do encanamento, sua equação é dada por

$$
P_{1}(t)-P_{2}(t)=g \bar{\rho}(t)\left(H_{2}+H_{3}\right)-\rho_{L} g h_{1}(t)
$$

onde: $P_{1}(t)$ é a pressão do gás na seção de alimentação do encanamento em $\mathrm{N} / \mathrm{m}^{2}$; $P_{2}(t)$ é a pressão do gás no topo do riser em $N / \mathrm{m}^{2} ; g$ é a gravidade $\left(9.81 \mathrm{~m} / \mathrm{s}^{2}\right) ; \bar{\rho}(t)$ é a densidade média da mistura no riser em $\mathrm{kg} / \mathrm{m}^{3} ; H_{2}$ é a altura do riser em $m$; $H_{3}$ é o diâmetro do riser em $m$; $\rho_{L}$ é a densidade do líquido considerada constante em $\mathrm{kg} / \mathrm{m}^{3} ; h_{1}(t)$ é o nível de líquido no declive em $m$.

O fluxo mássico da mistura $m_{m i x, o u t}(t)$ que sai do sistema encanamento-riser é determinado através da equação simplificada da válvula Z, dada por

$$
m_{\text {mix }, \text { out }}(t)=z K_{1} \sqrt{\rho_{T}(t)\left(P_{2}(t)-P_{G 1}(t)\right)}
$$

onde: $z$ é a abertura da válvula $(0-100 \%) ; K_{1}$ é um parâmetro de ajuste do modelo; $\rho_{T}(t)$ é a densidade na válvula em $\mathrm{kg} / \mathrm{m}^{3}$.

Observa-se que o acoplamento entre o sistema encanamento-riser e o separador ocorre através de uma relação de pressão ou seja, a pressão do gás $P_{G 1}(t)$ dentro do vaso é considerada, neste novo modelo, a pressão após a válvula $Z$ no topo do riser. Considerando o resultado apresentado na equação (2.6) é possível obter respectivamente o fluxo mássico de líquido

$$
m_{L, \text { out }}(t)=\alpha_{L}^{m}(t) m_{\text {mix }, \text { out }}(t)
$$

e o fluxo mássico de gás

$$
m_{G, \text { out }}(t)=\left[1-\alpha_{L}^{m}(t)\right] m_{m i x, \text { out }}(t)
$$

que saem através da válvula $Z$ no topo do riser, onde: $\alpha_{L}^{m}(t)$ é fração de líquido na válvula.

O fluxo mássico de líquido e de gás que saem do separador são representados respectivamente pela equação da válvula $V_{1}$ dada por

$$
m_{L S, \text { out }}(t)=z_{L} K_{4} \sqrt{\rho_{L}\left[P_{G 1}(t)+g \rho_{L} N(t)-P_{O L 2}\right]}
$$

onde: $z_{L}$ é a abertura da válvula $(0-100 \%) ; K_{4}$ é um parâmetro de ajuste do modelo; $P_{O L 2}$ é a pressão à jusante de $V_{1} \mathrm{em} N / \mathrm{m}^{2}$ e considerada constante. E pela equação da válvula $V_{2}$

$$
m_{G S, \text { out }}(t)=z_{G} K_{5} \sqrt{\rho_{G}(t)\left[P_{G 1}(t)-P_{G 2}\right]}
$$

onde: $z_{G}$ é a abertura da válvula $(0-100 \%) ; K_{5}$ é um parâmetro de ajuste do modelo; $\rho_{G}(t)$ é a densidade do gás em $\mathrm{kg} / \mathrm{m}^{3} ; P_{G 2}$ é a pressão à jusante da válvula $V_{2}$ em $N / m^{2}$ e considerada constante.

As condições de entrada na seção de alimentação do encanamento (i.e., $m_{L, \text { in }}$ e $\left.m_{G, i n}\right)$ são consideradas perturbações do processo e podem ser constantes, ou dependentes da pressão, nesse trabalho são assumidas constantes. A seguir é descrito como acontece a distribuição, e a velocidade das fases (gás-líquido) no sistema. Por fim, as equações internas, de transporte e geométricas para o modelo do sistema encanamento-riser apresentado nas equações (2.1)-(2.3) podem ser encontradas em $[6,7,8]$. 


\subsection{Deslocamento de gás no riser}

O deslocamento de gás ocorre através de uma relação entre o fluxo mássico de gás e a variação de pressão dentro da tubulação. Para a fase do gás negligenciam-se os termos de aceleração, então é a diferença de pressão que faz os fluidos escoarem tubulação acima, sua equação é dada por

$$
\Delta P(t)=P_{1}(t)-\left[P_{2}(t)+g \rho_{L} \alpha_{L}(t) H_{2}\right]
$$

onde: $\alpha_{L}(t)$ é a fração média de líquido no riser.

Observando a Figura 2 considera-se que existem duas situações no riser:

- $h_{1}(t)>H_{1}$ : nesse caso está ocorrendo a formação da golfada e o líquido está bloqueando o ponto-baixo, então tem-se que o fluxo mássico de gás interno no sistema encanamento-riser é $m_{\text {Gint }}(t)=0$, onde: $H_{1}$ é o nível de líquido crítico no ponto-baixo da tubulação em $m$.

- $h_{1}(t)<H_{1}$ : nesse caso o líquido não está bloqueando o ponto-baixo, então o gás fluirá do volume $V_{G 1}$ para o volume $V_{G 2}(t)$ com fluxo mássico de gás igual a $m_{\text {Gint }}(t)$.

Portanto, os dois principais parâmetros que determinam o deslocamento e a velocidade do gás no sistema são a variação de pressão $\Delta P(t)$ na tubulação, e a área livre no ponto-baixo dada pelo nível de líquido relativo $\left[H_{1}-h_{1}(t)\right] / H_{1}$. Então, é assumido que a variação de pressão move o gás através de uma válvula com a seguinte equação

$$
m_{\text {Gint }}(t)=K_{2} f\left(h_{1}(t)\right) \sqrt{\rho_{G 1}(t)\left[P_{1}(t)-P_{2}(t)-g \rho_{L} \alpha_{L}(t) H_{2}\right]}
$$

onde: $K_{2}$ é um parâmetro de ajuste do modelo; $f\left(h_{1}(t)\right)=\hat{A}(t)\left(H_{1}-h_{1}(t)\right) / h_{1}$ e $\hat{A}(t)$ é a área da seção transversal por onde passa o gás no ponto-baixo em $\mathrm{m}^{2}$; $\rho_{G 1}(t)$ é a densidade do gás no volume $V_{G 1} \mathrm{em} \mathrm{kg} / \mathrm{m}^{3}$.

O fluxo mássico de gás interno do volume $V_{G 1}$ para o volume $V_{G 2}(t)$ é dado por

$$
m_{\text {Gint }}(t)=v_{G 1}(t) \rho_{G 1}(t) \hat{A}(t)
$$

onde: $v_{G 1}(t)$ é a velocidade de gás no ponto-baixo em $\mathrm{m} / \mathrm{s}$. Por fim, substituindo a equação (2.10) na equação (2.9) e isolando $v_{G 1}(t)$ encontra-se que a velocidade do gás é

$$
v_{G 1}(t)=\left\{\begin{array}{cl}
K_{2} \frac{H_{1}-h_{1}(t)}{H_{1}} \sqrt{\frac{P_{1}(t)-P_{2}(t)-g \rho_{L} \alpha_{L}(t) H_{2}}{\rho_{G 1}(t)}} & \forall h_{1}(t)<H_{1}, \\
0 & \forall h_{1}(t) \geq H_{1} .
\end{array}\right.
$$

\subsection{Deslocamento de líquido no riser}

A distribuição de líquido ocorre através de uma equação de carregamento ou seja, considera-se que o gás carrega o líquido pelo riser, então modela-se diretamente a fração de volume de líquido $\alpha_{L T}(t)$ que sai através da válvula $\mathrm{Z}$ no topo do riser. Essa fração de líquido reside entre dois extremos: 
- $\alpha_{L T}(t)=\alpha_{L T}^{*}(t)$ : nesse momento o líquido bloqueia o ponto-baixo da tubulação e comporta-se como se estivesse em um tanque, por essa razão não há gás fluindo de $V_{G 1}$ para $V_{G 2}(t)\left(v_{G 1}(t)=0\right)$, onde $\alpha_{L T}^{*}(t)$ é a fração de líquido anterior a válvula $\mathrm{Z}$ no topo do riser sem entrada de gás, na maioria dos casos $\alpha_{L T}^{*}(t)=0$

- $\alpha_{L T}(t)=\alpha_{L}(t)$ : nesse momento a velocidade do gás é muito alta e a fração de líquido que sai no topo do riser é igual a fração de líquido no riser.

A transição entre esses dois extremos deve ser suave e ocorre da seguinte maneira: no instante em que o líquido bloqueia o ponto-baixo a fração de líquido no topo é $\alpha_{L T}^{*}(t)=0$, com o passar o tempo a quantidade de líquido no riser vai aumentando até que $\alpha_{L T}^{*}(t)>0$, nesse momento a pressão do gás na seção de alimentação é muito alta e conseqüentemente sua velocidade também, então ocorre o carregamento do líquido pelo riser e conseqüentemente para dentro do separador. É assumido que essa transição depende de um parâmetro $q(t)$ e que o carregamento de líquido é representado pela equação dada por

$$
\alpha_{L T}(t)=\alpha_{L T}^{*}(t)+\frac{q^{n}(t)}{1+q^{n}(t)}\left(\alpha_{L}(t)-\alpha_{L T}^{*}(t)\right)
$$

onde

$$
q(t)=\frac{K_{3} \rho_{G 1}(t) v_{G 1}^{2}(t)}{\rho_{L}-\rho_{G 1}(t)}
$$

e $K_{3}$ e $n$ são parâmetros de ajuste do modelo. Os detalhes da modelagem para a obtenção da equação (2.12) podem ser encontrados no trabalho de Storkaas et al. $[6,7,8]$.

\section{Resultados das Simulações e Discussões}

Nessa Seção são apresentados os resultados das simulações do modelo dinâmico simplificado para um sistema encanamento-riser-separador. Inicialmente são calculados os parâmetros de ajuste ou seja, $K_{1}$ na equação da válvula Z (2.6), $K_{2}$ na equação da velocidade do gás interna (2.11), $K_{3}$ e $n$ na equação de carregamento (2.12), $K_{4}$ na equação da válvula $V_{1}(2.7)$, e $K_{5}$ na equação da válvula $V_{2}(2.8)$. O cálculo desses parâmetros dependem de dados disponíveis de um sistema real ou provenientes de uma malha experimental, porém um conjunto completo desses dados não são encontrados na literatura, e também não são fornecido pelas indústrias de produção de petróleo. Para contornar esse problema no ajuste deste modelo utiliza-se um estudo de caso realizado por Storkaas [8] através do simulador de fluxo multifásico OLGA para o sistema encanamento-riser sob regime de fluxo com golfadas, onde a transição do regime de fluxo estável para um regime de fluxo com golfadas ocorre na abertura da válvula em $z_{\text {crit }}=13 \%$ denominada abertura crítica da válvula, em conjunto com um separador dimensionado a partir do trabalho de Miranda et al. [4] que possui as seguintes dimensões: comprimento igual a $C=4.5$ $m$, e diâmetro igual a $D_{s}=1.5 \mathrm{~m}$.

A tubulação do sistema encanamento-riser possui $0.12 m$ de diâmetro, alimentação de líquido e gás constantes e respectivamente iguais a $8.64 \mathrm{~kg} / \mathrm{s}$ e 0.362 


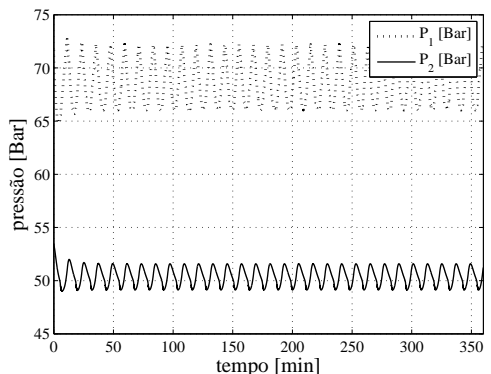

Figura 3: Pressões no sistema encanamento-riser com golfada.
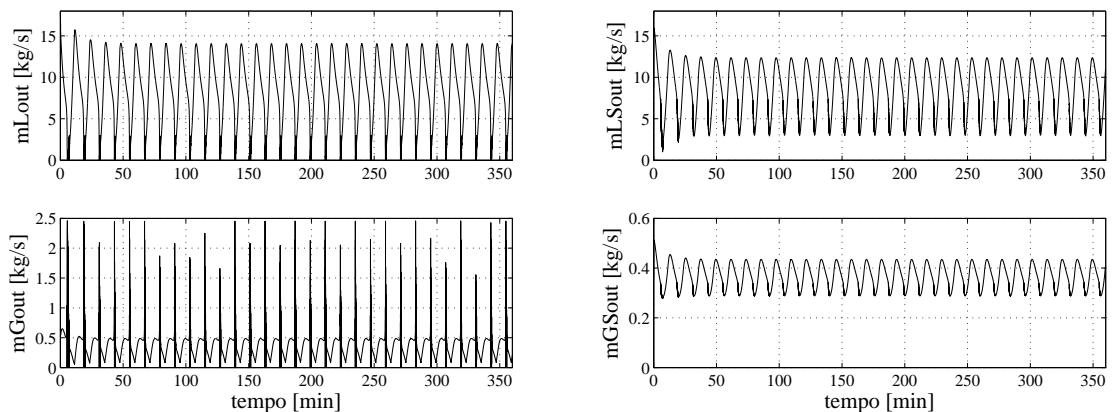

Figura 4: (Esquerda) Fluxos mássicos de gás e líquido que entram no separador. (Direita) Fluxos mássicos de gás e líquido que saem do separador.

$\mathrm{kg} / \mathrm{s}$. No início das simulações tem-se que o nível de líquido e a pressão do gás dentro do tanque são $N=0.75 \mathrm{~m}$ (nível de líquido na metade do tanque), e $P_{G 1}=50 \times 10^{5}$ $N / m^{2}$ (pressão usada no trabalho de Storkaas [8] após a válvula $Z$ no topo do riser). Os fluxos mássicos médios de líquido e gás que entram no separador são $8.64 \mathrm{~kg} / \mathrm{s}$ para o líquido, e $0.362 \mathrm{~kg} / \mathrm{s}$ para o gás (respectivamente os fluxos mássicos de líquido e gás que entram na seção de alimentação do sistema encanamento-riser). As válvulas $V_{1}$ e $V_{2}$ possuem abertura igual a $z_{L}=z_{G}=50 \%$ e são projetadas de modo que os fluxos mássicos na saída do separador quando as mesmas estão totalmente abertas $\left(z_{L}=z_{G}=100 \%\right)$ são iguais a $17.24 \mathrm{~kg} / \mathrm{s}$ e $0.724 \mathrm{~kg} / \mathrm{s}$ para o líquido e para o gás. As pressões após as válvulas $V_{1}$ e $V_{2}$ são constantes e escolhidas iguais a $P_{O L 2}=49 \times 10^{5} \mathrm{~N} / \mathrm{m}^{2}$ e $P_{G 2}=47.5 \times 10^{5} \mathrm{~N} / \mathrm{m}^{2}$. A partir disso são encontrados os seguintes parâmetros de ajuste para o modelo: $n=2.55, K_{1}=0.005$, $K_{2}=0.8619, K_{3}=1.2039, K_{4}=0.002$, e $K_{5}=0.0003$.

A seguir são apresentados os resultados das simulações considerando a abertura da válvula $\mathrm{Z}$ no topo do riser em $50 \%$. Na Figura 3 são apresentadas as oscilações de pressão na seção de alimentação do encanamento $P_{1}(t)$ e no topo do riser $P_{2}(t)$. $\mathrm{Na}$ Figura 4 observa-se a dinâmica dos fluxos mássicos de líquido e gás que entram (esquerda) e saem (direita) do separador. Observa-se que o regime de fluxo é oscilatório durante todo o intervalo de simulação caracterizando o regime de fluxo com golfadas. Nas Figura 5 e 6 são apresentados o nível de líquido e a pressão do gás dentro do separador observa-se que a golfada acontece entre um intervalo de tempo de aproximadamente $12 \mathrm{~min}$ dentro do vaso. 

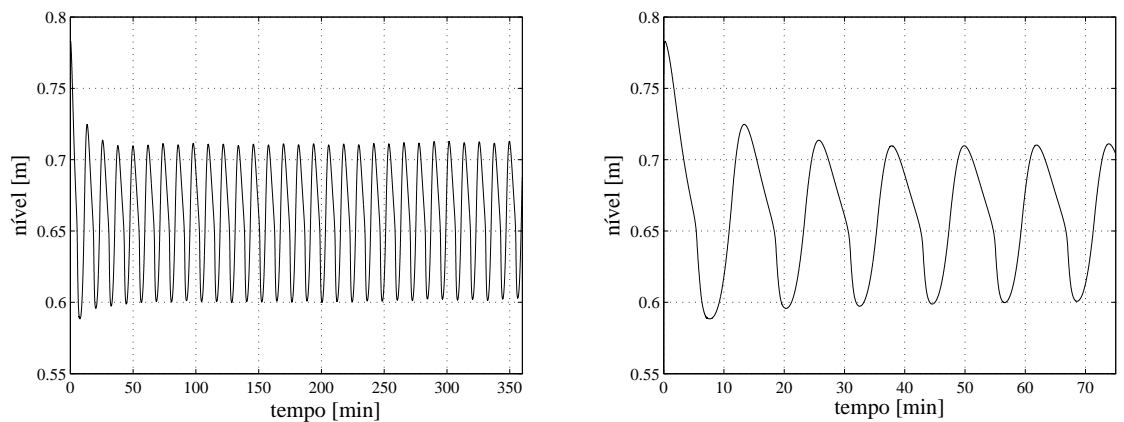

Figura 5: Nível de líquido dentro do separador.
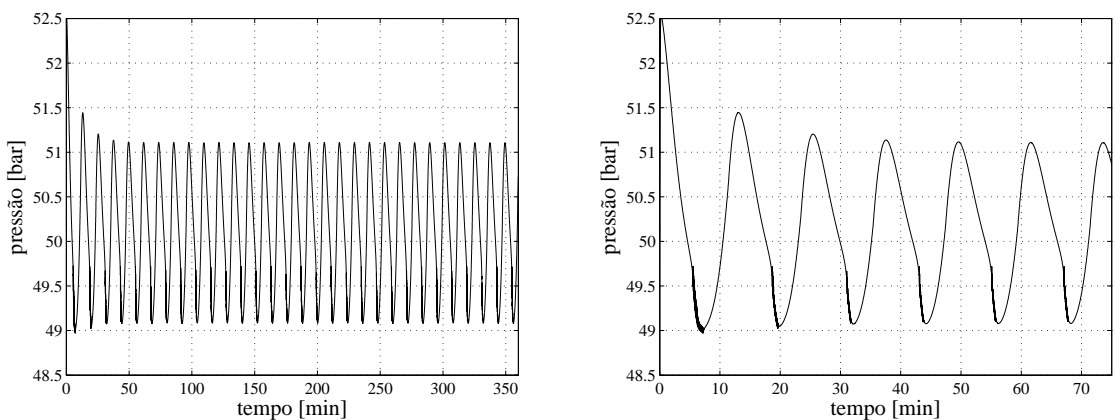

Figura 6: Pressão do gás dentro do separador.

\section{Conclusões}

Neste artigo é apresentado o desenvolvimento de um modelo dinâmico simplificado para um sistema encanamento-riser-separador com regime de fluxo bifásico (gás-líquido) sujeito ao escoamento com golfadas. O modelo resultante é formado pelo acoplamento entre o modelo dinâmico simplificado de Storkaas para o sistema encanamento-riser [8] e o modelo para o separador cilíndrico horizontal bifásico de Sausen e Barros [5]. Essa união ocorre através de uma relação de pressão ou seja, a pressão de gás dentro do tanque é considerada a pressão após a válvula $Z$ no topo do riser.

Foram realizados um número considerável de simulações e os resultados representaram de forma satisfatória a dinâmica da golfada no sistema encanamentoriser-separador. Como trabalhos futuros pretende-se utilizar o modelo proposto para definir e avaliar diferentes estratégias de controle para minimizar ou evitar a golfada em sistemas encanamento-riser-separador em indústrias de produção de petróleo.

Abstract. In this paper has been presented the development of a simplified dynamic model for a pipeline-riser-separator system under to the slug flow. This kind of outflow is characterized by severe and irregular flow with fluctuations of pressure in piping and in separation units in oil producing industries. The model obtained is the result of coupling between a simplified dynamic model system for the pipeline-riser in conditions of slug flow, and a model for a separator cylindrical 
horizontal biphase. Simulations results has shown that the model proposed represented a satisfactory way the slug in pipeline-riser-separator, as well as within of the tank.

\section{Referências}

[1] M.C.M. Campos, P. Laureiro, A.M. Borges Filho, Novas estratégias de controle para a plataforma de Petróleo P-55, em "Rio Oil e Gas Expo and Conference 2006", Rio de Janeiro, 2006.

[2] C.O. Essamas, "Numerical Modelling of Transient Gas-Liquid Flows Application to Stratified and Slug Flow Regimes", Tese de Doutorado, School of Engineering Applied Mathematics and Computing Group, Reino Unido, 2004.

[3] M.J. Godhavn, F.P. Mehrdad, P. Fuchs, New slug control strategies, tuning rules and experimental results, Journal of Process Control, 15 (2005), 547-577.

[4] M.A.A. Meira, A.M. Borges Filho, C.E.M. Vaz, Sistema de controle de golfadas, em "Rio Oil e Gas Expo and Conference 2004", Rio de Janeiro, 2004.

[5] A. Sausen, P.R. Barros, Modelagem matemática de um separador cilíndrico horizontal bifásico considerando um sistema encanamento-riser de fluxo com golfadas, em "7th Brazilian Conference on Dynamics, Control and Applications", pp. 117-133, Presidente Prudente, SP, 2008.

[6] E. Storkaas, S. Skogestad, J.M. Godhavn, Stabilization of severe slugging based on a low-dimensional nonlinear model, em "AIChE Annual Meeting", Paper 259e, Indianapolis, USA, 2002.

[7] E. Storkaas, S. Skogestad, A low-dimensional dynamic model of severe slugging for control design and analysis, em "11th International Conference on Multiphase Flow", pp. 117-133, San Remo, Italy, 2003.

[8] E. Storkaas, "Stabilizing Control and Controllability: Control Solutions to Avoid Slug Flow in Pipeline-Riser Systems", Tese de Doutorado, Norwegian University of Science and Technology, Norwegian, 2005. 